\title{
Impacto de la cobertura boscosa y uso de suelo sobre la textura y conductividad hidráulica del suelo en la subcuenca del río Estibaná
}

\author{
González, Alexandra \\ Facultad de Ingeniería Civil, Universidad Tecnológica de Panamá \\ Panamá, Panamá \\ alexandra.gonzalez1@utp.ac.pa \\ Rodríguez, Gerald \\ Facultad de Ingeniería Civil, Universidad Tecnológica de Panamá \\ Panamá, Panamá \\ gerald.rodriguez@utp.ac.pa

\section{Fábrega Duque, José} \\ Centro de Investigaciones Hidráulicas e Hidrotécnicas, Universidad Tecnológica de Panamá \\ Miembro del Sistema Nacional de Investigación de Panamá (SNI) \\ Panamá, Panamá \\ jose.fabrega@utp.ac.pa
}

\section{Abstract}

The sub-basin of the Estibana River is in the province of Los Santos, being the main tributary of the La Villa River. It is characterized by having an average rainfall of $1400 \mathrm{~mm} /$ year and decades of intense environmental degradation as a result of extensive agricultural activity. This situation make this sub-basin an attractive area to study the impact of these practices on soil texture and hydraulic conductivity. Hence, the objective of this work was to analyze these impacts through textural classes and infiltration estimation. The methodology consisted of taking soil samples in 54 points of the study area, to determine its texture by the Bouyoucos method. Likewise, estimates of the infiltration rate were made, using a Minidisk type infiltrometer. The resulting data were analyzed with computer tools such as $\mathrm{R}$ programs to obtain the texture triangle under the USDA parameters, the MACROS (hydraulic conductivity) and ArcGis (SIG) software. The textural classes with the greatest spatial distribution over the sub-basin were sandy loam (35.9\%) and loam (19.3\%). These results, according to the United States Soil Survey Division Staff, represent high (43\%) and moderately high (55\%) values. These values indicate soils with the capacity to fulfill their function of hydraulic conductivity. However, the bad use of soil and forest cover, can 
considerably reduce the areas with high conductivities, such as the middle part of the subbasin.

Keywords: Hydraulic Conductivity, Soil Texture, Land Use, Forest Cover, Agricultural.

\section{Resumen}

La subcuenca del río Estibaná, está ubicada en la provincia de Los Santos, siendo el principal afluente del río La Villa. Se caracteriza por tener precipitaciones promedio de $1400 \mathrm{~mm} /$ año y décadas de una intensa degradación ambiental producto de la actividad agropecuaria de forma extensiva, hacen de ésta, una zona atractiva para estudiar el impacto de estas prácticas sobre la textura de suelo y la conductividad hidráulica. De allí, que el objetivo del presente trabajo fue evaluar estos impactos a través de las clases texturales y estimación de infiltración. La metodología consistió en tomar muestras de suelo en 54 puntos del área de estudio, para la determinación de su textura por el método de Bouyoucos. Igualmente se realizaron estimaciones de tasa de infiltración, mediante la utilización de un infiltrómetro tipo Minidisk. Los datos resultantes fueron analizados con herramientas informáticas como programas en $R$ para la obtención del triángulo de textura bajo los parámetros del USDA, el software MACROS (conductividad hidráulica) y ArcGis (SIG). Las clases texturales con mayor distribución espacial sobre la subcuenca fueron: franco arenoso (35.9\%) y franco (19.3\%). Estos resultados, según el Soil Survey Division Staff de los Estados Unidos, representan valores altos (43\%) y moderadamente altos (55\%). Estos valores indican que los suelos encontrados poseen la capacidad de cumplir su función de conductividad hidráulica. Sin embargo, el mal uso de suelo y cobertura boscosa, puede reduce considerablemente las zonas con conductividades altas como lo es la parte media de la subcuenca.

Palabras claves: Conductividad Hidráulica, Textura de Suelo, Uso de Suelo, Cobertura Boscosa, Agropecuario.

\section{INTRODUCCIÓN}

El uso de suelo y la cobertura boscosa son indicadores que muestran la manera en que las sociedades manejan sus agroecosistemas. Esto puede contribuir a una conservación o degradación de suelo. Esta última puede ser por factores biofísicos como antrópicos, que pueden generar la destrucción masiva de coberturas vegetales, perdida de suelos, alteración a las propiedades físicas y extinción de aguas superficiales o acuíferos generando afectaciones sobre las poblaciones de la cual se abastecen. 
Dentro de la subcuenca del río Estibaná, la actividad agropecuaria intensiva ha devastado la cobertura boscosa y la condición de los suelos. Adicionalmente, las elevadas temperaturas y cambios en la precipitación por el cambio climático, ha dado lugar a sequias prolongadas. Como respuesta a esta situación, se ha optado por la construcción de pozos. Sin embargo, esta labor se ha desarrollado de forma desordenada, causando la sobreexplotación de las aguas subterráneas.

Para este estudio se evaluaron los impactos que generan el uso de suelo y la cobertura boscosa sobre las texturas de suelo y conductividad hidráulica encontradas en la subcuenca.

\section{MÉTODO}

\section{A. Pruebas de campo}

Consistieron en la realización de estimaciones de tasa de infiltración en 54 puntos del área de estudio, mediante la utilización de un infiltrómetro de tensión tipo Mini Disk [1]. Con este equipo se registró la variación de una columna de agua sobre el suelo analizado a intervalos constantes de tiempo, con succiones entre -3 y $-4 \mathrm{~cm}$. Igualmente, se tomaron muestras de suelo, entre 10 a $20 \mathrm{~cm}$ de profundidad.

\section{B. Pruebas de laboratorio}

Consistió en el análisis de hidrómetro de Bouyoucos $152 \mathrm{H}$, para determinar en porcentaje la distribución del tamaño de las partículas finas (arena, limo y arcilla) que pasan el tamiz $\mathrm{N}^{\circ} 200$.

\section{Análisis de resultados}

Se obtuvieron los resultados en porcentaje del contenido de arena, limo y arcilla de cada suelo que se sometió a las pruebas de hidrómetro utilizando el paquete de R Envalysis versión 0.4 [2]. Para establecer la clase textural del suelo según la clasificación de la USDA se utilizó el paquete Soil Texture versión 1.5.1 [3].

La conductividad hidráulica no saturada del suelo se obtuvo haciendo uso de MACROS de Excel suministrado por el fabricante del infiltrómetro Mini Disk. Finalmente, aplicando herramientas de sistemas de referenciación geográfica, en este caso ArcGIS Desktop 10.7.1 se crearon mapas de textura de suelo y conductividad hidráulica no saturada.

\section{RESULTADOS}

En la subcuenca del río Estibaná, se encontraron clases texturales: Franco arenoso, Arenoso franco, Franco, Franco arcilloso, Franco arcilloso limoso, Franco arcilloso arenoso y Arcilla. Sin embargo, las texturas franco arenoso (35.9\%) y franco (19.3\%) presentaron mayor distribución espacial sobre la subcuenca, especialmente, en la parte media y alta 
(figura 1). Generalmente, la subcuenca está representada por texturas gruesas (62\%) y medias (32\%).

Las texturas franco arenoso y franco arrojaron conductividades hidráulicas de 4.37 $\mathrm{cm} / \mathrm{h}$ y $2.5 \mathrm{~cm} / \mathrm{h}$, respectivamente. Estos resultados según el Soil Survey Division Staff de los Estados Unidos, representan valores altos (43\%) y moderadamente altos (55\%). Como muestra la figura 2, los valores más altos están localizados en la parte media de la subcuenca, haciendo de ella una zona importante para estudios más profundos.

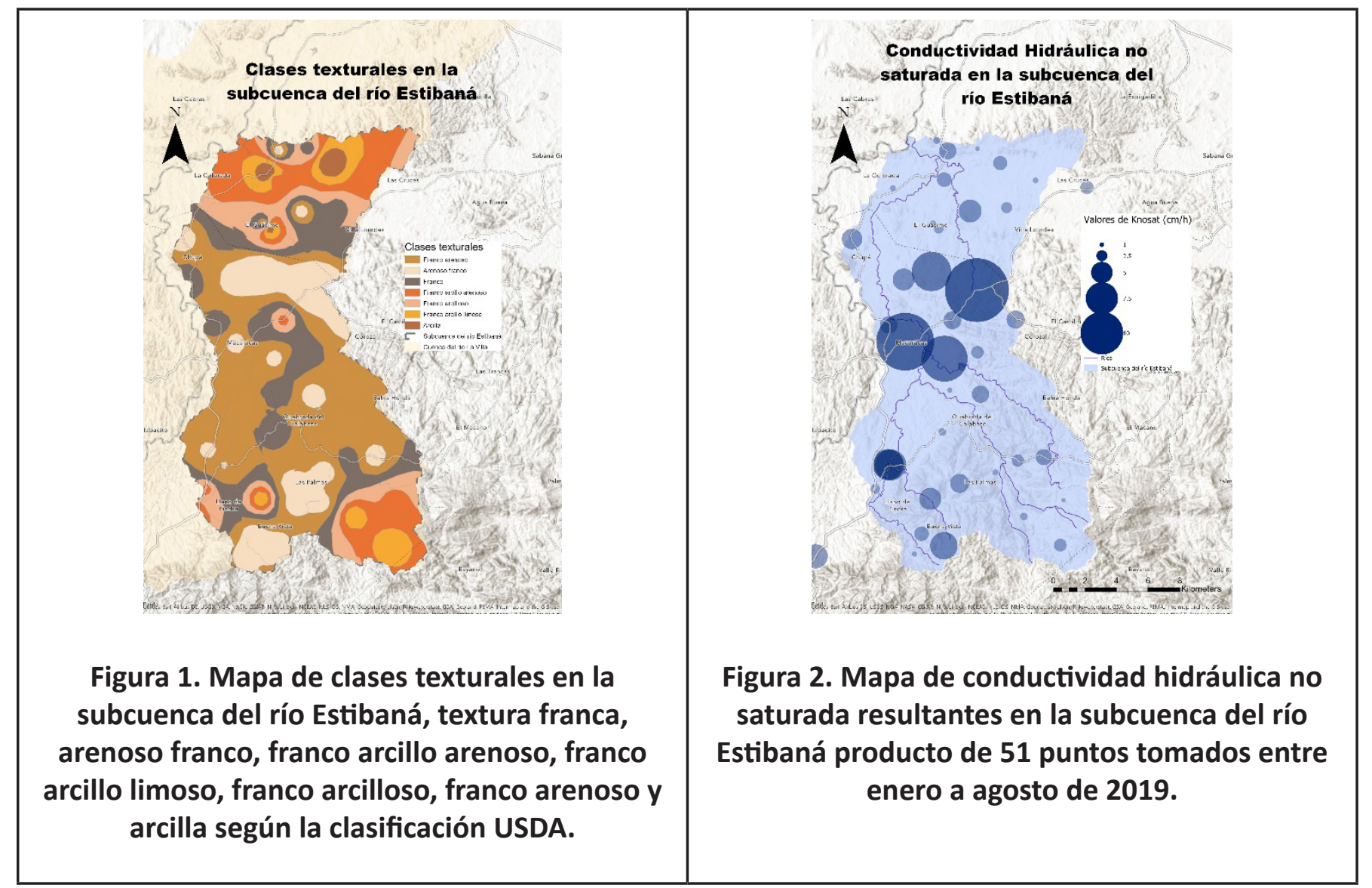

Las propiedades estudiadas, se ven afectadas por el uso de suelo y la cobertura boscosa. En la figura 3 se muestra que el $67.5 \%$ de la capacidad agrológica de la subcuenca corresponde a un uso de suelo tipo VII, exclusivo para uso forestal [4] [5] . Contrario a esto, el mapa de cobertura boscosa (figura 4) muestra un $83.4 \%$ destinado a pasto y uso agropecuario. Por otro lado, la carga ganadera (UG/Ha unidad de ganado por hectárea) para ecosistemas frágiles no debe exceder de $1 \mathrm{UG} / \mathrm{Ha}$. Sin embargo, para esta subcuenca es de $1.5 \mathrm{UG} / \mathrm{Ha}$ [6]. Consecuentemente, existe un alto grado de compactación sobre los suelos de textura gruesa, en especial sobre las texturas franco arenosa [7]. La superficie descubierta y compactada, incrementa la erosión y los niveles de escorrentía que a su vez 
degradan los suelos al perder su capa de materia orgánica. Igualmente, hay una disminución de la infiltración reduciendo la recarga de aguas subterráneas, con mayor énfasis en la parte media de la subcuenca. Por último, según el balance hídrico elaborado para el área de estudio, el $66 \%$ de la precipitación se pierde por evapotranspiración producto de los pastos [8].

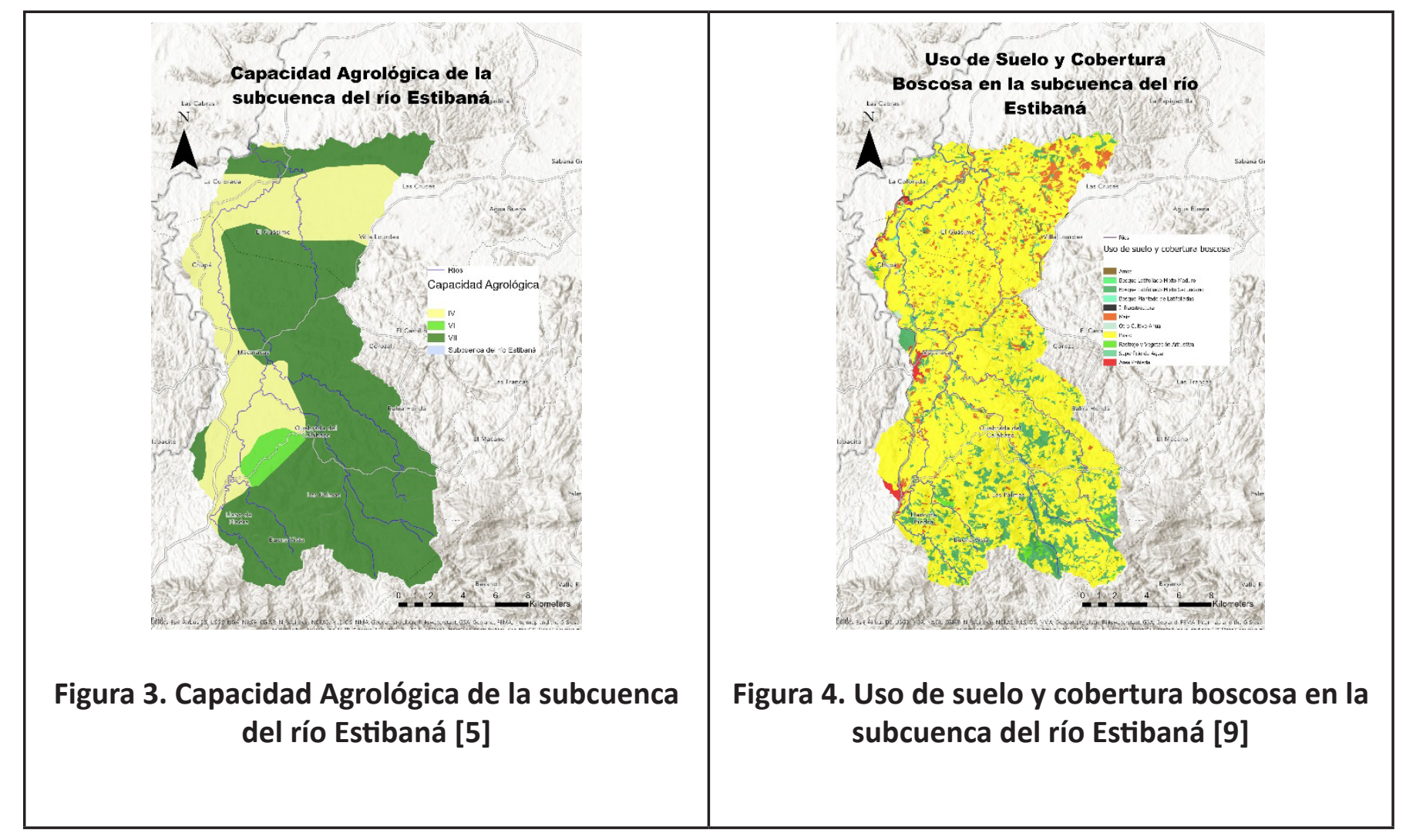

\section{CONCLUSIONES}

La subcuenca presenta suelos de textura gruesa que poseen la capacidad de cumplir su función de conductividad hidráulica, en especial en la parte media de la subcuenca, considerándose está, como una posible zona de recarga. Sin embargo, la mala gestión en cuanto a la capacidad agrológica de los suelos, destinada a uso de pastos y actividad agropecuaria, donde el pisoteo provocado por el sobrepastoreo y la deforestación afectan las propiedades físicas estudiadas, pueden reducir considerablemente las zonas con conductividades altas, además de alterar el ciclo hidrológico y la perdida de suelo. Es necesario la ejecución de un plan de ordenamiento orientado a la capacidad agrológica de la subcuenca, para cuidar los suelos y el agua subterránea a largo plazo. 


\section{Referencias}

[1] I. Decagon Devices, “Mini Disk Infiltrometer," 2016. doi: https://doi.org/10.3929/ethz-b-000238666.

[2] Z. Steinmets, "Package 'envalysis' Miscellaneous Functions for Environmental Analyses." Apr. 17, 2020, doi: 10.1016/0016.

[3] J. Moeys et al., "Package 'soiltexture' Functions for Soil Texture Plot, Classification and Transformation." Sep. 19, 2018, Accessed: Oct. 11, 2020. [Online]. Available: https://github.com/ julienmoeys/soiltexture.

[4] J. E. Villareal, "Caracterización de los suelos de la Provincia de Herrera," no. Suelos, 2018, doi: 10.13140/RG.2.2.18861.33763.

[5] CONADES, "Estrategia provincial de desarrollo sostenible de Los Santos," Panamá, 2014.

[6] V. Opolenko, "Sensibilidad medioambiental a la desertificación en la cuenca del río La Villa, República de Panamá," Universidad Internacional de Andalucía, Huelva, 2014.

[7] A. Hossne, Y. Mayorca, L. Salazar, F. Subero, and A. Zacillo, "Humedad compactante y sus implicaciones agrícolas en dos suelos franco arenoso de sabana del estado Monagas, Venezuela," Rev. Científica UDO Agrícola, vol. 9, no. 4, pp. 937-950, 2009, Accessed: Jul. 10, 2021. [Online]. Available: http://www.bioline.org.br/request?cg09110.

[8] Z. Rodríguez, "Balance hídrico de la subcuenca del río Estibaná," Universidad Tecnológica de Panamá, 2021.

[9] MIAMBIENTE, “Mapa de cobertura y uso de la tierra 2012," 2015.

\section{Autorización y Licencia CC}

Los autores autorizan a APANAC XVIII a publicar el artículo en las actas de la conferencia en Acceso Abierto (Open Access) en diversos formatos digitales (PDF, HTML, EPUB) e integrarlos en diversas plataformas online como repositorios y bases de datos bajo la licencia CC: Attribution-NonCommercial-ShareAlike 4.0 International (CC BY-NC-SA 4.0) https://creativecommons. org/licenses/by-nc-sa/4.0/.

Ni APANAC XVIII ni los editores son responsables ni del contenido ni de las implicaciones de lo expresado en el artículo. 